\section{Magnetic and structural properties of $\mathrm{Sc}\left(\mathrm{Fe}_{1-x} S \mathrm{i}_{x}\right)_{2}$ Laves phases studied by Mössbauer spectroscopy and neutron diffraction}

\author{
Marek Wiertel, \\ Zbigniew Surowiec, \\ Mieczysław Budzyński, \\ Jan Sarzyński, \\ Anatoly I. Beskrovnyi
}

\begin{abstract}
The aim of the presented paper is to study an influence of replacement of Fe atoms by Si atoms in quasibinary $\mathrm{Sc}\left(\mathrm{Fe}_{1-x} \mathrm{Si}_{x}\right)_{2}$ Laves phases on their structural and magnetic properties. Powder X-ray diffraction (XRD) and neutron diffraction (ND) measurements carried out at different temperatures from $4.3 \mathrm{~K}$ up to about $700 \mathrm{~K}$ revealed that samples were single phase with cubic $C 15$ structure for Si concentration $x$ from 0.05 to 0.20 and hexagonal $C 14$ structure for higher concentration. The results of ${ }^{57} \mathrm{Fe}$ Mössbauer measurements showed that the $\mathrm{Sc}\left(\mathrm{Fe}_{1-x} \mathrm{Si}_{x}\right)_{2}$ compounds with $x \leq 0.30$ are ferrimagnetic at $4.3 \mathrm{~K}$. At temperature $80 \mathrm{~K}$ in the samples with $x=0.20$ and 0.30 , a magnetic cluster spin-glass state has been observed, as ferrimagnetic long-range order disappears. Such picture was supported by the results of ND measurements carried out at 8 K, which confirmed the lack of long-range order for $x$ above 0.10 and an occurrence of hyperfine field distributions in the corresponding Mössbauer spectra. At room temperature, samples with $x \geq 0.20$ became paramagnetic. A substitution of Si atoms for Fe ones leads to a decreasing of mean values of hyperfine magnetic fields in samples under investigation. From the neutron diffraction pattern analysis of $\mathrm{Sc}\left(\mathrm{Fe}_{0.90} \mathrm{Si}_{0.10}\right){ }_{2} \mathrm{Fe}$ magnetic moment was determined as to be equal to $1.5 \mu_{\mathrm{B}}$ at $8 \mathrm{~K}$. Combining this result with a value of hyperfine magnetic field on ${ }^{57} \mathrm{Fe}$ probes, the hyperfine coupling constant $\mathrm{A}$ in $\mathrm{Sc}\left(\mathrm{Fe}_{0.90} \mathrm{Cu}_{0.10}\right)_{2}$ phases is estimated at about $11.6 \mathrm{~T} / \mu_{\mathrm{B}}$ at $8 \mathrm{~K}$.
\end{abstract}

Key words: hyperfine fields • Mössbauer effect • neutron diffraction • quasibinary Laves phase

M. Wiertel ${ }^{\bowtie}$, Z. Surowiec, M. Budzyński, J. Sarzyński Institute of Physics,

M. Curie-Skłodowska University,

1. M. Curie-Skłodowskiej Sq., 20-031 Lublin, Poland,

Tel.: +48 81537 6220, Fax: +48 815376191 ,

E-mail: marek.wiertel@umcs.pl

A. I. Beskrovnyi

Frank Laboratory of Neutron Physics Joint Institute for Nuclear Research,

141980 Dubna, Russia

Received: 18 June 2014

Accepted: 5 November 2014

\section{Introduction}

For long-time issues concerning the onset of magnetism in concentrated substitutional ternary alloys was a matter of interest [1]. In particular, such type compounds belonging to pseudobinary $\mathrm{A}\left(\mathrm{Fe}_{1-x} \mathrm{M}_{x}\right)_{2}$ Laves-phase class reveal a large diversity of magnetic state varying from ferro- or antiferromagnetism via a spin cluster and spin-glass regime to the paramagnetic state for most of the Fe replacing elements, being either a metal or a metalloid (e.g. Al, Si) [2]. A few common features of investigated materials strongly affect their magnetic behavior. The first of them is the triangular in-plane lattice symmetry, which leads to a higher coordination number of Fe atoms $(z=6)$ and potentially to magnetic topological frustrations, cluster spin-glass and spin glass state. The second essential factor is chemical and crystallographic disorder as a result $\mathrm{M} / \mathrm{Fe}$ substitution. These factors can be the origin of the spin or cluster spin-glass magnetic ordering. The next crucial property of investigated compounds is a shape of their $d$-electrons local density of states (LDOS) curves. Due to strong $3 d-3 d$ hybridization, LDOS consists of many sharp and narrow peaks near the Fermi level [3]. The latter feature stimulates rather a strong influence of substitution of $\mathrm{M}$ atoms for $\mathrm{Fe}$ ones on 
the magnetic properties of the investigated samples. In the quasibinary system $\mathrm{Sc}\left(\mathrm{Fe}_{1-x} \mathrm{Si}_{x}\right)_{2}$, the starting compound $\mathrm{ScFe}_{2}$ crystallizes in the hexagonal $C 36$ Laves-phase structure and it is a weak itinerant electron ferrimagnet with $T_{\mathrm{C}}=653(5) \mathrm{K}$ [4]. According to the Sc-Fe-Si ternary diagram, members of quasibinary system with $0.02 \leq x \leq 0.04$ have a cubic $C 15$ structure. In the concentration range between 0.04 and 0.17 , the cubic $C 15$ and hexagonal $C 14$ structures coexist. For the concentration $x \geq 0.17$, the stable pure hexagonal $C 14$ phase occurs [5]. On the other hand, an existence of $\mathrm{ScSi}_{2}$ compound theoretically proposed in [6] has not been univocally experimentally confirmed up to now. In the $\mathrm{ScFe}_{2}$, Fe atoms occupy three non-equivalent positions: $6 g, 6 h$ and $4 f$ (space group $P 6_{3} / m m c$ ) and have magnetic moments equaling 1.31(1), 1.78(1), and $1.52(1) \mu_{\mathrm{B}}$ at room temperature (RT), respectively. The mean value of hyperfine magnetic field in this compound amounts to 18.2(2) T [7]. In the regular cubic $C 15$ structure, Fe atoms occupy only one type of available sites: $16 d$ (space group $F d \overline{3} \mathrm{~m}$ ) with the point symmetry $\overline{3} \mathrm{~m}$ and form regular tetrahedrons connected via their corners. Six Fe and Sc ( $8 a$ positions) atoms surround each $\mathrm{Fe}$ atom composing its nearest neighborhood. The investigated compounds showing ferrimagnetic properties belong to the class of materials called itinerant magnets [8]. One of the important characteristics of these materials is that the amplitude of the local spin fluctuation varies significantly with temperature, [9], or with local concentration fluctuations.

The aim of the presented study was to examine the influence of Si substitution for Fe on structural and magnetic properties of $\mathrm{Sc}\left(\mathrm{Fe}_{1-x} \mathrm{Si}_{x}\right)_{2}$ compounds for selected concentrations. In particular, dependence of hyperfine interaction parameters, $T_{\mathrm{C}}$ and Fe magnetic moments on concentration $x$ were of special interest to us.

\section{Experimental details}

Intermetallic compounds of $\mathrm{Sc}\left(\mathrm{Fe}_{1-x} \mathrm{Si}_{x}\right)_{2}$ for $x \leq$ 0.30 in the polycrystalline form were prepared by arc melting stoichiometric amounts of high-purity scandium, iron and copper under an inert argon atmosphere. The ingots were annealed in vacuum at about $1100 \mathrm{~K}$ for 72 hours to ensure their homogeneity. The crystallographic structures of the samples were examined by means of X-ray powder diffraction with $\mathrm{CuK}_{\alpha}$ radiation. The XRD patterns were obtained in the approximate $2 \theta$ range of $33-80^{\circ}$ in steps of $0.03^{\circ}$. The Mössbauer spectra of powdered samples were recorded at $4.3 \mathrm{~K}, 80 \mathrm{~K}$ and room temperature using a constant acceleration spectrometer and ${ }^{57} \mathrm{Co}(\mathrm{Rh})$ source. Two different physical models were used to fit experimental spectra: (i) discrete model in which the number of subspectra and their relative contributions were determined on the basis of crystallographic information on Fe sites and number of local surroundings of them originating from binomial distribution of Si atoms, respectively, and (ii) hyperfine field distribution model for $x \geq$
0.20 and spectra measured at $80 \mathrm{~K}$. The obtained spectra were generally analyzed under the assumption that the energy of the quadrupole interaction is small compared to the hyperfine magnetic splitting $\left(e Q V_{z z} / 4 \mu B_{\mathrm{hf}}<<1\right)$. In the discrete set of sextets model, only the nearest neighbors were taken into account because merely replacement of the Fe atom in near neighborhood has significant influence and may be registered with satisfactory reliability. The solving of static Hamiltonian calculations for mixed magnetic and quadrupole interactions procedure was used in cases in which this assumption could not have been applicable. The isomer shift (IS) values are given in relation to $\alpha$-Fe at room temperature.

For the samples with $x=0.10,0.20$, and 0.30 , neutron powder diffraction measurements were carried out in the temperature range from 8 to $700 \mathrm{~K}$. The DN2 time-of-flight diffractometer at the fast-pulsed reactor IBR2 in the Frank Laboratory of Neutron Physics, Joint Institute for Nuclear Research was used. The $\mathrm{X}$-ray and neutron diffraction patterns were analyzed using the FULLPROF program based on the Rietveld method. A convolution pseudo-Voigt with double exponential function was chosen to generate the line shape of the diffraction peaks. In the final run, the following parameters were refined from the XRD and ND data: scale factor, unit cell parameters, positional coordinates of atoms, thermal factors and additionally for ND magnitude and orientation of the Fe and Sc ferrimagnetically ordered magnetic moments. The neutron coherent scattering lengths for $\mathrm{Sc}, \mathrm{Fe}$, and $\mathrm{Al}$ were taken as equal to $12.3 \mathrm{fm}, 9.45 \mathrm{fm}$, and $7.72 \mathrm{fm}$, respectively. In the ND patterns of $\mathrm{Sc}\left(\mathrm{Fe}_{1-x} \mathrm{Si}_{x}\right)_{2}$, one pair of (111) and (101) peaks at $d \approx 4.05 \AA$ with the strong dependence of their intensity on temperature was found (see Fig. 5). For these peaks, nuclear scattering contributions from different atoms at different crystallographic sites cancel each other out to a large extent and have almost purely magnetic character. This fact allows us to determine the magnitude of the $\mathrm{Fe}$ and Sc ordered magnetic moments.

\section{Results and discussion}

The obtained XRD patterns of the samples with indicated concentration $x$ are shown in Fig. 1. The lattice parameter $a$ of the cubic phases obey Vegard's law and only slightly decreases from 7.0340(3) $\AA$ for $x=0.05$ to $7.0236(6) \AA$ for $x=0.20$ (see middle panel on left side of Fig. 1). Small admixtures of $\mathrm{Sc}_{2} \mathrm{O}_{3}$ (below 3-5\%) were detected in ND measurements (see Fig. 5).

Mössbauer measurements performed at $4.3 \mathrm{~K}$ revealed that all investigated samples are ferromagnetic. All observed magnetic sextets are slightly asymmetric. In the regular cubic $C 15$ phase, $\mathrm{Fe} / \mathrm{Si}$ atoms occupy only one type of site: $16 d$ with the point symmetry $\overline{3} \mathrm{~m}$. However, there are two magnetically non-equivalent types of $\mathrm{Fe}$ positions in this structure for which an easy magnetization axis $<111>$ makes the angles $\theta$ of $70^{\circ} 32^{\prime}$ (site Type I) or $0^{\circ}$ (site Type II) with the principal axis of the electric field gradient. A population ratio of these sites is equal 


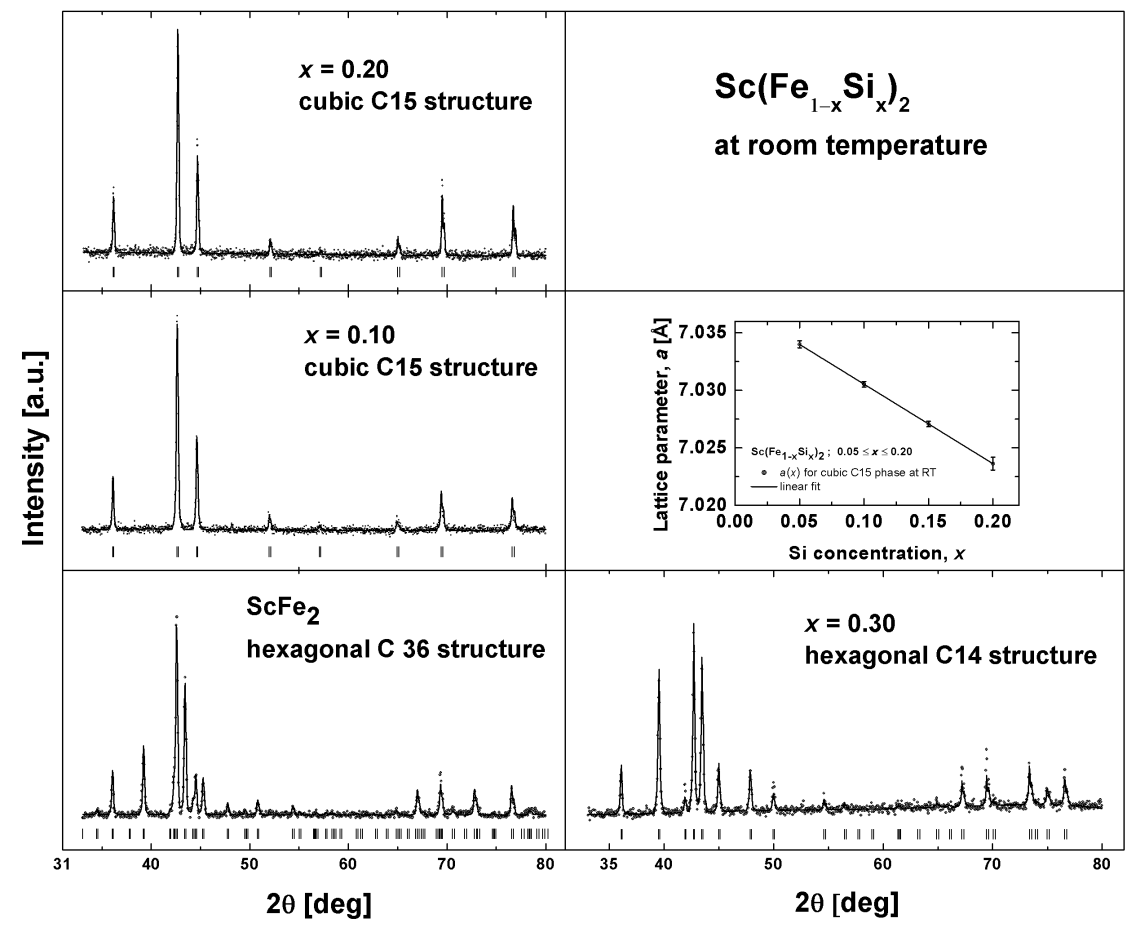

Fig. 1. XRD patterns of $\mathrm{Sc}\left(\mathrm{Fe}_{1-x} \mathrm{Si}_{x}\right)_{2}$ samples for the indicated concentrations $x$.

to $3: 1$, respectively. In such a case, two different mean quadrupole shifts $\varepsilon_{\mathrm{Q}, \mathrm{I}}$ and $\varepsilon_{\mathrm{Q}, \mathrm{II}}$ (where $\varepsilon_{\mathrm{Q}}=$ $\mathrm{QS}\left(3 \cos ^{2} \theta-1\right) / 2$ and the ratio $\varepsilon_{\mathrm{Q}, \mathrm{I}} / \varepsilon_{\mathrm{Q}, \mathrm{II}}$ is equal to $-1: 3)$ for sextet components corresponding to those are expected. Such situation is valid for all investigated at different temperatures quasibinary cubic phases in which ferromagnetic order exists. It means that the direction of the easy magnetization axis is not changed when Fe atoms are replaced with

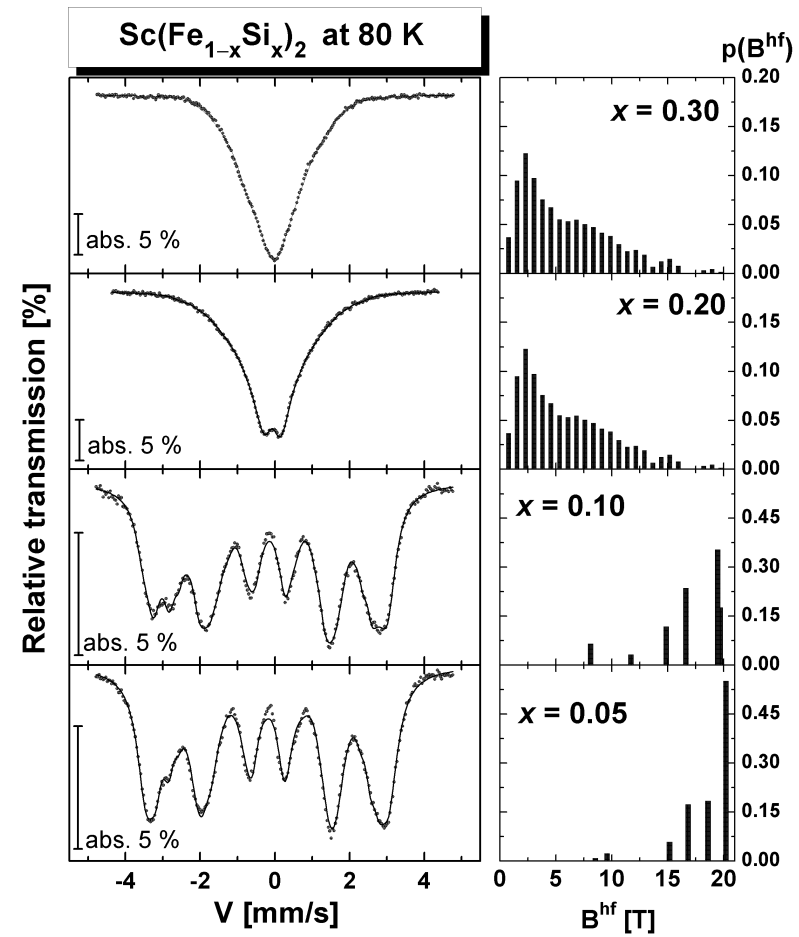

Fig. 2. The fitted ${ }^{57} \mathrm{Fe}$ Mössbauer spectra of $\mathrm{Sc}\left(\mathrm{Fe}_{1-x} \mathrm{Si}_{x}\right)_{2}$ measured at liquid nitrogen temperature (left) and the derived discrete or quasi-continuous hyperfine field distributions (right) of $\mathrm{Sc}\left(\mathrm{Fe}_{1-x} \mathrm{Si}_{x}\right)_{2}(0.05 \leq x \leq 0.3)$ compounds.
$\mathrm{Si}$ ones. For the hexagonal $\mathrm{Sc}\left(\mathrm{Fe}_{0.70} \mathrm{Si}_{0.30}\right)_{2}$ sample, $\mathrm{Fe} / \mathrm{Si}$ atoms occupy two kinds of crystallographic sites: $6 h$ and $2 a$ with the point symmetries $m m 2$ and $\overline{3} m$, respectively. In this structure, the population ratio of the sites is also equal to $3: 1$ but angles between an easy magnetization axis $<001>$ and local principal axes of the electric field gradient are equal to $90^{\circ}$ ( $6 h$ site) or $0^{\circ}(2 a$ site $)$. It gives a ratio of $\varepsilon_{\mathrm{Q}, 6 h}$ and $\varepsilon_{\mathrm{Q}, 2 a}$ values $-1: 2$. In the fitting procedure performed, the population ratios of the sites and corresponding quadrupole shift value ratios for all sextets were fixed.

Figure 2 shows the Mössbauer spectra of $\mathrm{Sc}\left(\mathrm{Fe}_{1-x} \mathrm{Si}_{x}\right)_{2}$ in the $0.05 \leq x \leq 0.30$ ranges measured at $80 \mathrm{~K}$. The right panel of Fig. 2 shows the contributions of sextets of intensity larger than $5 \%$ for samples with $x=0.05$ and 0.10 where discrete statistical model is applicable. In the case of two samples with higher $x$, a good fit could be obtained only by fitting hyperfine field distributions $p\left(B_{\mathrm{hf}}\right)$. A linear correlation between IS and hyperfine magnetic field was assumed. When the temperature increases, the shape of the spectra changes toward the one that is largely influenced by relaxation phenomena. Hence it should be necessary to take into account both phenomena, that is, relaxation and $B_{\mathrm{hf}}$ distribution. The form of spectra at $80 \mathrm{~K}$ point to the existence of magnetic clusters with the distribution of relaxation times, evolving with decreasing temperature. An exact value of the freezing temperature corresponding to the time constant of the Mössbauer experiment $\left(\tau \approx 2 \times 10^{-8} \mathrm{~s}\right)$ cannot be deduced but the blocking temperatures of the different clusters should be distributed around freezing temperature $T_{\mathrm{fc}}$. Formation of magnetic clusters and micromagnetic behavior at low temperatures were observed earlier in analogical pseudobinary phases many times [10-12]. For the sample with higher $x$, the $p\left(B_{\mathrm{hf}}\right)$ distribu- 


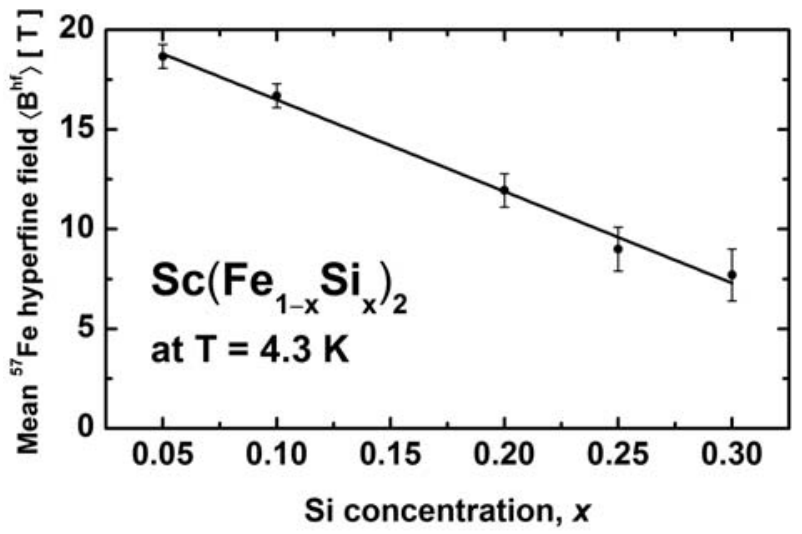

Fig. 3. The dependence of the mean ${ }^{57} \mathrm{Fe}$ hyperfine magnetic fields, $<B_{\mathrm{hf}}>$, at $4.3 \mathrm{~K}$ on Si concentration.

tion curves generally are composed of roughly two broad Gaussian peaks centered around $\sim 2.3 \mathrm{~T}$ and $\sim 6.6 \mathrm{~T}$ for $\mathrm{Sc}\left(\mathrm{Fe}_{0.80} \mathrm{Si}_{0.20}\right)_{2}$ and $\sim 2.6 \mathrm{~T}$ and $\sim 6.5 \mathrm{~T}$ for $\mathrm{Sc}\left(\mathrm{Fe}_{0.70} \mathrm{Si}_{0.30}\right)_{2}$ alloys. Their standard deviations are equal to approximately 1.9 and $7.3 \mathrm{~T}$, and 3.5 and $6.2 \mathrm{~T}$, respectively. The latter has approximately two times greater area than the former for $x=0.20$, and 1.5 times greater for $x=0.30$. Such form of hyperfine field distributions suggests non-uniform character of the cluster spin-glass ordering being, to some extent, reflection of two different types of $\mathrm{Fe}$ crystallographic sites. In order to check a tendency to change the freezing temperature of spin clusters $T_{\mathrm{fc}}$, the additional Mössbauer measurements were performed for the $\mathrm{Sc}\left(\mathrm{Fe}_{0.85} \mathrm{Si}_{0.15}\right)_{2}$ sample. At room temperature, the respective spectrum (not shown here) was very similar to the spectra for samples showing spin clustering and has hyperfine field distribution with the average value of $\left\langle B_{\mathrm{hf}}\right\rangle=$ $8.36(19) \mathrm{T}$. This result proves that $T_{\mathrm{fc}}$ have tended to increase with decreasing extent of substitution of $\mathrm{Si}$ atoms for Fe ones in the studied samples.

At room temperature the samples with $x \geq 0.20$ are paramagnetic. Their Mössbauer spectra were fitted by means of discrete sets of doublet components, of which the number and relative contributions result from binominal distribution for the given $x$ value.

The calculated mean values ${ }^{57} \mathrm{Fe}$ hyperfine magnetic field, $B_{\mathrm{hf}}$, at $4.3 \mathrm{~K}$ are shown in Fig. 3 as a function of Si concentration. This dependence has linear character with a slope of about $-46 \mathrm{~T}$ per Si atom. Moreover, from detailed analysis of hyperfine magnetic fields values for individual local surroundings, it results that the substitution of one $\mathrm{Si}$ atom for $\mathrm{Fe}$ atom in the nearest neighborhood of ${ }^{57} \mathrm{Fe}$ nuclear probes decreases the mean hyperfine magnetic field by about $3.5 \mathrm{~T}$.

In the investigated samples, nearly axially symmetric electric field gradients give average values of quadrupole splitting (QS) changing from $-0.496(10) \mathrm{mm} / \mathrm{s}$ for $x=0.05$ to $-0.320(10) \mathrm{mm} / \mathrm{s}$ for $x=0.30$.

In Fig. 4, the mean IS dependence on the concentration at room temperature is given. IS increases almost linearly from about $-0.19(1) \mathrm{mm} / \mathrm{s}$ for $\mathrm{ScFe}_{2}$ up to $-0.04(1) \mathrm{mm} / \mathrm{s}$ for the $\mathrm{Sc}\left(\mathrm{Fe}_{0.70} \mathrm{Si}_{0.30}\right)_{2}$ sample. This increase is the largest one of among other $\mathrm{M}$ atom for which measurements had been carried out

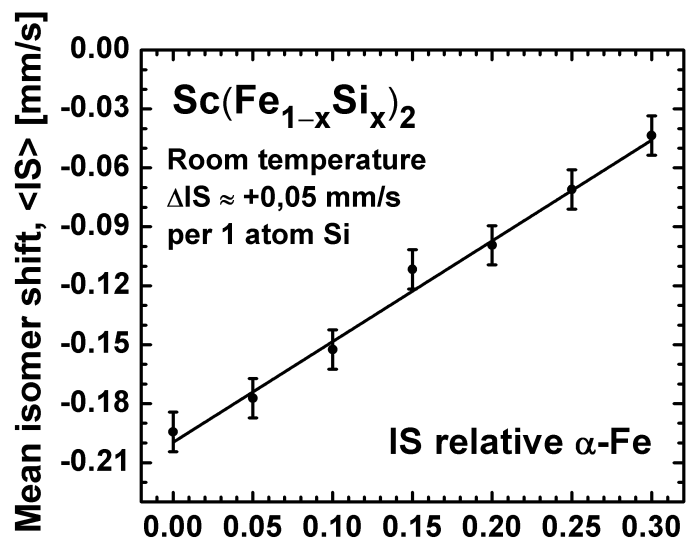

Si concentration, $x$

Fig. 4. Mean isomer shift dependence on Si concentration $x$ at room temperature.

and is due to, mainly, a large electronegativity of $\mathrm{Si}$ (1.74 in Allred Rochow scale). If the calibration of the observed IS in terms of total $s$-electron density given in the paper [13] is used, it can be derived, based on IS change, that the replacement of $\mathrm{Fe}$ by $30 \% \mathrm{Si}$ reduces the average $s$-electrons charge at the nuclear probe ${ }^{57} \mathrm{Fe}$ by about 0.1 electrons.

The neutron diffraction (ND) patterns of $\mathrm{Sc}\left(\mathrm{Fe}_{1-x} \mathrm{Si}_{x}\right)_{2} \quad(x=0,0.1,0.2$ and 0.3$)$ recorded at $8 \mathrm{~K}$ are shown in Fig. 5. For sample with $x=0.10$

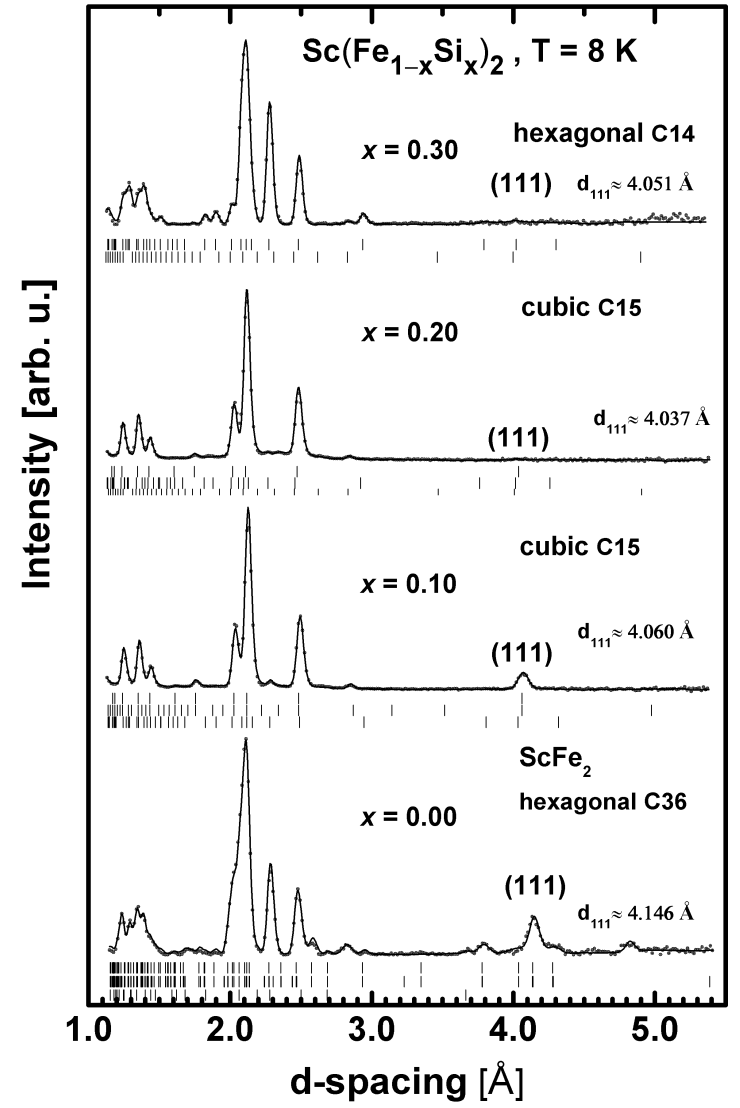

Fig. 5. ND patterns of $\mathrm{Sc}\left(\mathrm{Fe}_{1-x} \mathrm{Si}_{x}\right)$ for $x=0.10,0.20$ and 0.30 at $8 \mathrm{~K}$. The points represent experimental data, the full lines show calculated pattern. The short vertical marks below the diffraction pattern for $x=0.10$ indicate the calculated nuclear Bragg peak positions (the upper-most row) and the magnetic ones (lower row) for quasibinary phase. The third row shows nuclear Bragg peaks position for $\mathrm{Sc}_{2} \mathrm{O}_{3}$. 


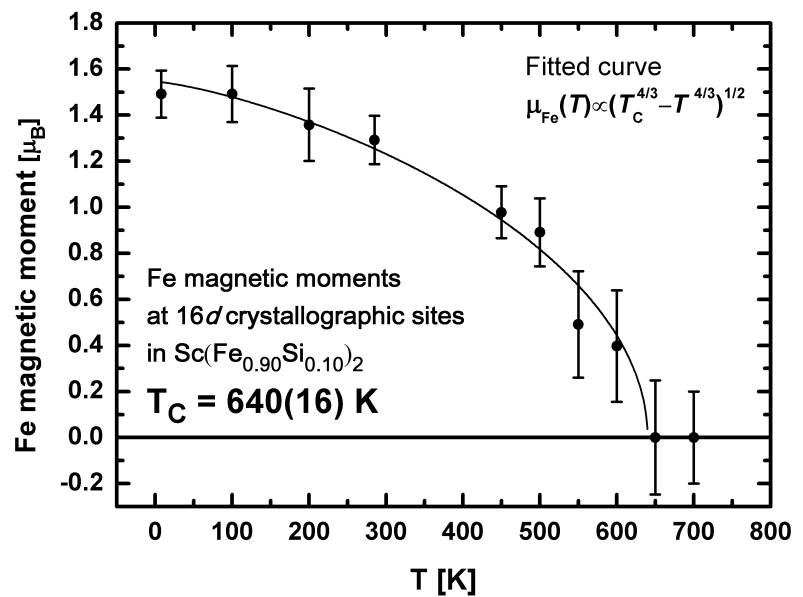

Fig. 6. The temperature dependence of Fe magnetic moments obtained from ND patterns for the $\mathrm{Sc}\left(\mathrm{Fe}_{0.90} \mathrm{Si}_{0.10}\right)_{2}$ sample. The solid line represents a least-square fit of the expression predicted in the framework of SCR theory of spin fluctuations [9]

ND measurements in the wide range of temperature from 8 to about $700 \mathrm{~K}$ have been performed. On the basis of these measurements, mean Fe magnetic moments and their temperature dependences were determined. Curie temperature $T_{\mathrm{C}}$ was estimated from the fitting to experimental data a curve resulting from self-consistent renormalized (SCR) theory of spin fluctuations [9] (see Fig. 6). The values of $T_{\mathrm{C}}$ for pure $\mathrm{ScFe}_{2}$ and the quasibinary samples as a function of $x$ are shown in Fig. 7. The points for $x=$ 0.06 and 0.25 are taken from a paper [14] in which $T_{\mathrm{C}}$ is determined from ac-susceptibility measurements. Curie temperature monotonically decreases from $650(5) \mathrm{K}$ for $\mathrm{ScFe}_{2}$ to about $90 \mathrm{~K}$ for $x=0.30$.

Thanks to the use of two independent methods Mössbauer spectroscopy (MS) and neutron diffraction (ND) to determine magnetic moments of Fe atoms and ${ }^{57} \mathrm{Fe}$ hyperfine fields in the $\mathrm{Sc}\left(\mathrm{Fe}_{0.90} \mathrm{Si}_{0.10}\right)_{2}$ Laves phase it was possible to estimate a hyperfine coupling constant A. The obtained value of about

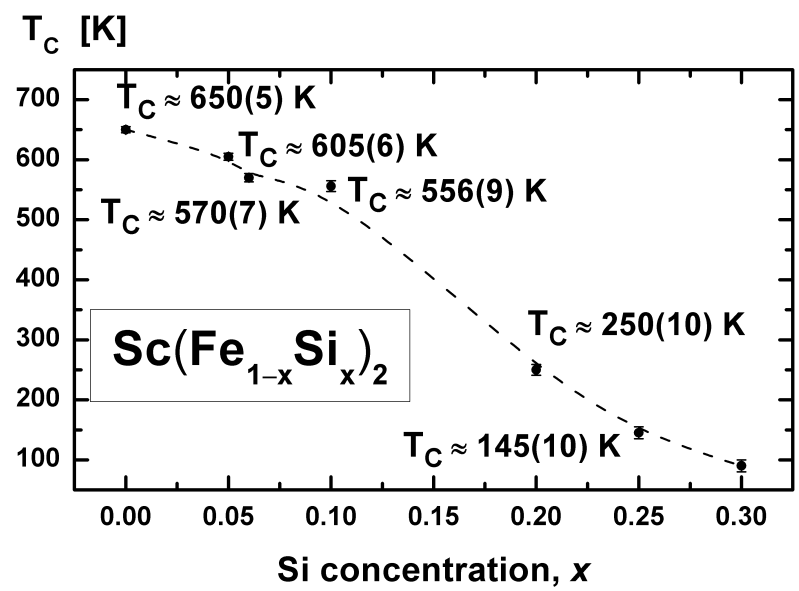

Fig. 7. The variation of the Curie temperature $T_{\mathrm{C}}$ values in $\mathrm{Sc}\left(\mathrm{Fe}_{1-x} \mathrm{Si}_{x}\right)_{2}$ series determined by extrapolation of the SCR theory curve fitted to the ${ }^{57} \mathrm{Fe}$ mean hyperfine magnetic fields temperature dependences. The $T_{\mathrm{C}}$ temperatures for samples with $x=0.06$ and 0.25 are taken from a paper [14] in which $T_{\mathrm{C}}$ are determined from ac-susceptibility measurements. The dashed line is a guide for eyes.
11.6(1.2) $\mathrm{T} / \mu_{\mathrm{B}}$ at $8 \mathrm{~K}$ is in the limits of error independent on temperature.

\section{Conclusions}

It is possible to obtain a single-phase quasibinary $\mathrm{Sc}\left(\mathrm{Fe}_{1-x} \mathrm{Si}_{x}\right)_{2}$ Laves phases by a conventional arc-melting method. Presented XRD and ND studies confirmed a sequence of the structural changes from $C 36$ type structure for $\mathrm{ScFe}_{2}$ via $C 15$ type for $0.05 \leq x \leq 0.20$ to $C 14$ type in $\mathrm{Sc}\left(\mathrm{Fe}_{0.70} \mathrm{Si}_{0.30}\right)_{2}$. In the $\mathrm{Sc}\left(\mathrm{Fe}_{1-x} \mathrm{Si}_{x}\right)_{2}$ samples with $x \leq 0.10$, direct first-order transition from ferromagnetic to paramagnetic state occurs. In contrast, for samples with $x=0.20$ and 0.30 , an intermediate cluster spin-glass state is revealed between ferromagnetic and paramagnetic states. Short-range exchange frustrations caused by a random $\mathrm{Si} / \mathrm{Fe}$ substitutions together with the triangular in-plane lattice symmetry are the reasons leading to such kind of magnetic state in non-diluted magnets. The lack of a long-range magnetic order down to $8 \mathrm{~K}$ was concluded from neutron diffraction measurements. Unfortunately, due to high background, it was impossible to obtain from our ND measurements any information on neutron diffuse scattering and the magnetic short-range order.

The linear decrease of the average hyperfine magnetic field with increasing $x$ in $\mathrm{Sc}\left(\mathrm{Fe}_{1-x} \mathrm{Si}_{x}\right)_{2}$ may be qualitatively explained by the increase of $3 d$ band occupation at Fermi level.

\section{References}

1. Hilscher, G. (1982). Onset of magnetism in concentrated ternary alloys I. J. Magn. Magn. Mater., 25(3), 229-250. DOI: 10.1016/0304-8853(82)90071-3.

2. Hilscher, G. (1982). Onset of magnetism in concentrated ternary alloys I. Laves phase compounds $\mathrm{A}\left(\mathrm{Fe}_{1-x} \mathrm{~B}_{x}\right)_{2}(\mathrm{~A}=\mathrm{Y}, \mathrm{Zr}, \mathrm{U} ; \mathrm{B}=\mathrm{Mn}, \mathrm{Co}$ and $\mathrm{Al})$. J. Magn. Magn. Mater., 27(1), 1-31. DOI: 10.1016/03048853(82)90279-7.

3. Al Alam, A. F., Matar, S. F., Ouaini, N., \& Nakhl, M. (April 2008). Hydrogen insertion effects on the magnetic properties and chemical bonding within C14 Laves phases. Prog. Solid State Chem., 36(3), 192-212. DOI: 10.1016/j.progsolidstchem.2008.07.001.

4. Pokatilov, V. S., Sadchikov, V. V., Sidokhin, E. F., \& Utenkova, O. V. (1986). Structure and magnetic properties of Fe-Sc alloys. Fiz. Met. Metalloved., 62(1), 69-75.

5. Gladyshevskii, E. I., Kotur, B. Y., Bodak, V. P., \& Skvorchuk, V. P. (1977). Scandium-iron-silicon system. Dopov. Akad. Nauk Ukr. RSR, A, Fiz.-Tekh. Mat. Nauki (Ukrainian SSR), 39(8), 751-754.

6. Niessen, A. K., \& DeBoer, F. R. (1981) The enthalpy of formation of solid borides, carbides, nitrides, silicides, and phosphides of transition and noble metals. J. Less-Common Met., 82, 75-80. DOI: 10.1016/00225088(81)90200-9.

7. Wiertel, M., Surowiec, Z., Beskrovnyi, A. I., Sarzyński, J., \& Budzyński, M. (2005). Determination of magnetic moments and hyperfine fields in $\mathrm{ScFe}_{2}$ Laves phase compound. Annual Report Frank Laboratory of Neutron Physics. Dubna, Russian Federation: Joint 
Institute for Nuclear Research. Retrieved June 18, 2014 from: http://flnp.jinr.ru/img/304/188_2005_Annual_Report.pdf.

8. Yamada, H. (1988). Electronic structure and magnetic properties of the cubic Laves phase transition metal compounds. Physica B+C, 149(1/3), 390-402. DOI: 10.1016/0378-4363(88)90270-7.

9. Moriya, T. (1982). Spin fluctuations in ferromagnetic metals - temperature variation of local moment and short range order. J. Phys. Soc. Jpn., 51 (2), 420-434. DOI: http://dx.doi.org/10.1143/JPSI.51.420.

10. Muraoka, Y., Shiga, M., \& Nakamura, Y. (1979) Magnetic properties and Mössbauer effects of $\mathrm{Zr}\left(\mathrm{Fe}_{1-\mathrm{x}} \mathrm{Co}_{\mathrm{x}}\right)_{2}$.J.Phys. F-Metal Phys., 9(9), 1889-1904. DOI: 10.1088/0305-4608/9/9/019.

11. Reissner, R., Steiner, W. (1986). Electrostatic hyperfine interactions in $\mathrm{Y}\left(\mathrm{Fe}_{1-x} \mathrm{Al}_{x}\right)_{2}$. Hyperfine Interact., 28(1/4), 1017-1020. DOI: 10.1007/BF02061617.
12. Sarzyński, J., Budzyński, M., Wasiewicz, R., \& Wiertel, M. (1992). The influence of silicon on hyperfine magnetic fields in $\mathrm{Zr}\left(\mathrm{Fe}_{1-x} \mathrm{Si}_{x}\right)_{2}$ measured for $x \leq 0.17$ J. Phys.-Condens. Matter, 4(30), 6473-6478. DOI: 10.1088/0953-8984/4/30/014.

13. Walker, L. R., Wertheim, G. K., \& Jaccarino, V. (1961). Interpretation of the ${ }^{57} \mathrm{Fe}$ isomer shift. Phys. Rev. Lett., 6(3), 98-101. DOI: http://dx.doi.org/10.1103/ PhysRevLett.6.98.

14. Kotur, B. Y., Gratz, E., Bauer, E., Hilscher, G., Kottar, A., Michor, H., Reichl, Ch., Wiesinger, G., \& Markosyan, A. S. (1998). Magnetic and electrical properties of $\mathrm{Sc}\left(\mathrm{M}_{1-x} \mathrm{Si}_{x}\right)_{2-y}(\mathrm{M}=\mathrm{Fe}, \mathrm{Co}, \mathrm{Ni})$. J. Alloy. Compd., 278(1/2), 49-59. DOI: 10.1016/S09258388(98)00642-2. 